\title{
Role of fast ion pressure in the isotope effect in JET L-mode plasmas
}

\section{JET Contributors}

2019-09

\section{JET Contributors, Hatch , D R, Ahlgren , T, Aho-Mantila , L , Airila , M , Björkas , C ,} Heinola , K, Lahtinen , A, Nordlund , K, Safi , E, Pehkonen , S-P , Salmi , A , Tala , T \& Bonanomi , N 2019 , ' Role of fast ion pressure in the isotope effect in JET L-mode plasmas ' , Nuclear Fusion , vol. 59 , no. 9 , 096030 . https://doi.org/10.1088/1741-4326/ab2d4f

http://hdl.handle.net/10138/324312

https://doi.org/10.1088/1741-4326/ab2d4f

acceptedVersion

Downloaded from Helda, University of Helsinki institutional repository.

This is an electronic reprint of the original article.

This reprint may differ from the original in pagination and typographic detail.

Please cite the original version. 


\title{
Role of fast ion pressure in the isotope effect in JET L-mode plasmas
}

\author{
N. Bonanomi ${ }^{1}$, I. Casiraghi ${ }^{2,3}$, P. Mantica $^{3}$, C. Challis ${ }^{4}$,

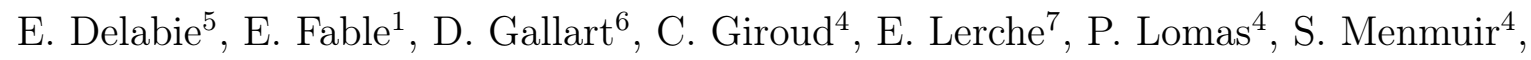 \\ G. M. Staebler ${ }^{8}$, D. Taylor ${ }^{4}$, D. Van Eester ${ }^{7}$, and JET contributors*
}

EUROfusion Consortium, JET, Culham Science Centre, Abingdon, OX14 3DB, UK

1) Max Planck Institute for Plasma Physics, Boltzmannstr. 2, 85748 Garching, Germany

2) University of Milano-Bicocca, Milano, Italy

3) CNR - Plasma Physics Institute "P. Caldirola", Milano, Italy

4) Culham Centre for Fusion Energy, Abingdon, OX14 3DB, UK

5) Oak Ridge National Laboratory, P.O. Box 2008 Oak Ridge TN 37831 , USA

6) Barcelona Supercomputing Center, Barcelona, Spain

7) LPP-ERM/KMS, TEC partner, Brussels, Belgium

8) General Atomics, P.O. Box 85608, San Diego, California 92186-5608, USA

*See the author list of "Overview of the JET preparation for Deuterium-Tritium Operation" by E. Joffrin et al. to be published in Nuclear Fusion Special issue: overview and summary reports from the 27th Fusion Energy Conference (Ahmedabad, India, 22-27 October 2018)

\begin{abstract}
This paper presents results of JET ILW L-mode experiments in hydrogen $(H)$ and deuterium $(D)$ plasmas, dedicated to the study of the isotope dependence of ion heat transport by determination of the ion critical gradient and stiffness by varying the ICRH power deposition. When no strong role of fast ions in the plasma core is expected, the main difference between the two isotope plasmas is determined by the plasma edge and the core behavior is consistent with a gyro-Bohm scaling. When the heating power (and the fast ion pressure) is increased, in addition to the difference in the edge region, also the plasma core shows substantial changes. The stabilization of ion heat transport by fast ions, clearly visible in $D$ plasmas, appears to be weaker in $H$ plasmas, resulting in a higher ion heat flux in $H$ with apparent anti-gyro-Bohm mass scaling. The difference is found to be caused by the different fast ion pressure between $H$ and $D$ plasmas, related to the heating power settings and to the different fast ion slowing down time, and is completely accounted for in non-linear gyrokinetic simulations. The application of the TGLF quasi-linear model to this set of data is also discussed.
\end{abstract}




\section{Introduction}

Differences in the energy confinement time of hydrogen $(H)$ and deuterium $(D)$ plasmas have been observed in many devices since the first comparisons between the performances of different hydrogen isotope plasmas $[1,2,3,4]$. Besides the confinement time, also other fundamental aspects for a future reactor, such as the L-H power transition $[1,4,5]$ and the $\mathrm{H}$-mode pedestal $[1,4,6]$, have been found to substantially change with the isotope mass. Early theoretical expectations based on ITG (Ion Temperature Gradient) instabilities within an adiabatic electron, electrostatic and collision-less approximation pointed to the idea that turbulent transport should follow the gyro-Bohm mass scaling (turbulent transport $\propto \sqrt{m_{i}}$ ) [7]. However, experimentally, heavier isotopes have generally been found to have better confinement time and performances $[1,3,4,8]$. This apparent contrast between theory and experimental observations is generally referred as 'isotope effect' and has been the focus for many research works in recent time $[8,9,10]$. In reality, many effects can break the simplistic gyro-Bohm mass dependence and cause the observed behavior of the turbulent transport. Kinetic electrons [11], electromagnetic effects [9, 12], collisions [10], differences in the zonal flows [13], critical gradient threshold differences, $E \times B$ flow shear $[9,14]$ and also differences in sources and sinks can all cause deviations from the gyro-Bohm scaling with the mass. Understanding the behavior of turbulent transport with the isotope mass is essential for predicting future reactors, ITER (International Thermonuclear Experimental Reactor) in first place. Fusion power production is pursued in a mixture of isotope gases $(D T)$ and the first operations of ITER will be in $H$ or $H e$ plasmas [15]. An uncertainty therefore still exists on predicted performances due to an incomplete knowledge on the effects of isotope mass on confinement, which prevents safe extrapolation to ITER from present results in $D$ plasmas and also in perspective from the first ITER operational phases in $H$ or $H e$ to the next phases in $D$ and $D T$. A clear understanding of the relation between turbulent transport and isotope mass is then highly desirable. This has motivated an intense experimental effort on JET (Joint European Torus) to investigate the mass effects from $H$ to $D$ to $T$, with dedicated campaigns in $D$ and $H$ and one in $T$ foreseen in early 2020.

Amongst the experiments repeated in $H$ and $D$ (and scheduled in $\mathrm{T}$ ), the one addressed in this paper concerns the determination of the core ion critical gradient and stiffness level in L-mode plasmas by using ion heat flux scans at approximately constant temperature by applying ICRH power on- and off-axis in $\left({ }^{3} \mathrm{He}\right)-\mathrm{D}$ scheme for strong and localized thermal ion heating [16]. We present the experimental results in a set of $H$ and $D$ L-mode plasmas with similar densities, geometry, plasma current, magnetic field and heating power, focusing on the core turbulent ion heat transport and particularly on the relation between normalized ion heat flux and normalized ion temperature gradient $\left(R / L_{T i}=R\left|\nabla T_{i}\right| / T_{i}\right)$, that can be extracted from this kind of experiments. This approach provides more insight into the transport mechanisms and is more powerful for model validation than the more common profile comparison studies. In this case, it has allowed us to identify the role of the fast ion pressure as a potential cause of breaking of the gyro-Bohm scaling, in addition to the others mentioned above.

The paper is organized as follows: in section 2 the experimental set-up is described and in section 3 the numerical simulation set-up is presented. In section 4 the experimental results are described, while in section 5 the gyrokinetic and the quasi-linear simulations results are shown and compared to the experiment. The conclusions are reported in section 6 .

\section{Experimental set-up and methods}

The plasmas studied in this work are JET tokamak (major radius $R_{0}=2.96 \mathrm{~m}$, minor radius $a=1 \mathrm{~m}$ ) L-mode $D$ plasmas with carbon wall (CW) and $H$ and $D$ plasmas with the ITER-like wall (ILW). The discharges have similar plasma shape, vacuum toroidal magnetic field $B_{T}=3.2 \pm 0.1 T$, plasma current $I_{p} \approx 2 M A$, electron 
density on the magnetic axis $n_{e, 0} \sim 3.5 \pm 0.5 \cdot 10^{19} \mathrm{~m}^{-3}$, safety factor at the flux surface that contains the $95 \%$ of the poloidal flux $q_{95} \approx 5$ and similar total heating power. Radio frequency (RF) heating power consists of ICRH heating mainly deposited on thermal ions in minority scheme (3He)-D [16] using a concentration $n_{3} \mathrm{He}_{e} / n_{e} \approx 6 \%$ in $D$ plasmas and $n_{3} \mathrm{He} / n_{e} \approx 2 \%$ in $H$ plasmas. NBI power have also been applied using $\sim 70 \mathrm{kV}$ ion beams in $H$ plasmas and $\sim 100 \mathrm{kV}$ ion beams in $D$ plasmas. Different levels of ICRH power (2.5-7 MW) and of NBI power $(1.5-8 \mathrm{MW})$ have been used in different discharges in order to obtain a wider variation of the ion heat flux and of $L_{T i}^{-1}\left(=\left|\nabla T_{i}\right| / T_{i}\right)$ and in order to study the effect of fast ions on the thermal transport in the plasma core [17]. The ICRH power deposition and the ${ }^{3} \mathrm{He}$ fast ion density and energy density have been evaluated using the PION code $[18,19]$ or, in some cases, the SELFO code $[20,21]$. The NBI power deposition on electrons and on ions and the NBI fast ion pressure have been calculated with the PENCIL code [22]. The ohmic power density has been calculated analytically as $p_{O h m}=\eta \cdot j^{2}$, where $\eta$ is the Spitzer plasma resistivity and $j$ is the plasma current density reconstructed by the EFIT equilibrium code [23]. Also the exchanged power density between ions and electron, $p_{e i}$, has been calculated analytically (for the definitions used to calculate $p_{O h m}$ and $p_{e i}$ see ref. [24]). The radiated power density $p_{\text {rad }}$ has been estimated from bolometric measurements. The powers $P_{O H M}, P_{I C R H}, P_{N B I}, P_{e i}$ and $P_{r a d}$ have been obtained integrating over the plasma volume and the ion and electron heat fluxes are expressed in gyro-Bohm units as

$$
\begin{aligned}
q_{i, g B} & =\left[\left(P_{I C R H, i}+P_{N B I, i}+P_{e i}\right) / \Sigma\right] \cdot R_{0}^{2} /\left(n_{e} T_{i} \rho_{i}^{2} v_{t h, i}\right) \\
q_{e, g B} & =\left[\left(P_{I C R H, e}+P_{N B I, e}+P_{O H M}-P_{e i}-P_{r a d}\right) / \Sigma\right] \cdot R_{0}^{2} /\left(n_{e} T_{e} \rho_{s}^{2} c_{s}\right)
\end{aligned}
$$

where $\Sigma$ is the area of the considered flux surface, $c_{s}=\sqrt{T_{e} / M_{i}}, v_{t h, i}=\sqrt{T_{i} / M_{i}}, \rho_{i}=v_{t h, i} M_{i} / e B_{r e f}$ and $\rho_{s}=c_{s} M_{i} / e B_{r e f}, M_{i}$ being the plasma main ion mass $(H$ or $D)$ and $B_{r e f}$ the value of the magnetic field at the center of the considered flux surface. Typical errors on the heat fluxes are of the order of $20 \%$. For the measurement of the electron temperature $\left(T_{e}\right)$ the ECE (Electron Cyclotron Emission) and the high-resolution Thomson scattering (HRTS) diagnostics have been used. The ion temperature $T_{i}$ and the plasma toroidal rotation $\omega_{T}$ have been measured by the active Charge Exchange (CX) diagnostic using the signals from plasma impurities ( $C$ for the $\mathrm{CW}$ discharges $N e$ for the ILW discharges and also $B e$ for shots n. 90671) and, for the ILW plasmas, a charge-exchange measurement, recently made available at JET, that uses beam modulation for background subtraction and fits all active line features to extract the deuterium charge exchange line [25, 26]. The electron density $n_{e}$ has been measured using the HRTS diagnostic. The safety factor $q$, the magnetic shear $s=r / q \cdot d q / d r$ and the equilibrium plasma geometry have been reconstructed by the EFIT equilibrium code with Faraday rotation or Motional Stark Effect constraints, depending on the availability. Typical error bars of these quantities are $\sim 15-20 \%$. Local values of $R / L_{T i}=-R_{0} \nabla T_{i} / T_{i}, R / L_{T e}=-R_{0} \nabla T_{e} / T_{e}$ and $R / L_{n}=-R_{0} \nabla n_{e} / n_{e}$ are obtained by local linear fits of $\ln \left(T_{i}\right), \ln \left(T_{e}\right)$ and $\ln \left(n_{e}\right)$ radial profiles. The fits are done using $r=\left(R_{\text {out }}-R_{\text {in }}\right) / 2, R_{\text {out }}$ and $R_{\text {in }}$ being the outer and inner major radii of the flux surface on the magnetic axis plane, and averaging other multiple fits using a variable number of data points around the chosen radius (usually 3-9 points). Errors on these quantities are typically $\sim 15-20 \%$. The data analysis has been carried out mainly at the toroidal radius $\rho_{\text {tor }}=0.33$, where $\rho_{\text {tor }}=\sqrt{\Phi / \Phi_{\max }}, \Phi$ being the toroidal magnetic flux, corresponding to $\rho_{\text {pol }} \sim 0.42, R \sim 3.3 \mathrm{~m}, \mathrm{r} / \mathrm{a} \sim 0.33$. The chosen radial position and the time- intervals considered ensured optimal conditions for studying the effect of fast ions on the thermal transport while avoiding magnetohydrodynamic instabilities or other large-scale instabilities. The electron density and temperature and the safety factor and magnetic shear of ILW discharges n. 90673 and n. 91710 (high NBI power $D$ and $H$ ) are shown in Fig. 1. The electron temperature of discharges n. 90671 and n. 91626 (low NBI power $D$ and $H$ ) is also shown in Fig. 1, while the electron density and safety factor for these discharges are very similar to the previous ones and are not shown for more clarity in the plots. 


\section{$3 \quad$ Numerical simulation set-up}

The characteristics of the turbulent transport in the plasma and the turbulent component of the heat fluxes have been calculated through linear and non-linear gyrokinetic simulations using the GENE (Gyrokinetic Electromagnetic Numerical Experiment) code [27, 28]. GENE solves the gyrokinetic 5D Vlasov equations [29, 30] coupled with the Maxwell equations within a $\delta f$ approximation using field aligned coordinates $\{x, y, z\}$, where $z$ is the coordinate along the magnetic field line, $x$ is the radial coordinate and $y$ is the binormal coordinate.

All the simulations are performed in the local limit, using Miller geometry [31] as well as collisions, external flow shear $\gamma_{E \times B}$, kinetic ions, kinetic electrons and, in some cases, kinetic fast ions ( $H$ or $D$ fast ions from NBI and ${ }^{3} \mathrm{He}$ fast ions from ICRH). Finite- $\beta$ effects (considering both $B_{\perp}$ and $B_{\|}$fluctuations) have also been considered. For ${ }^{3} \mathrm{He}$ and NBI fast ions, an equivalent Maxwellian distribution function has been used. It is important to remind that, when considering highly energetic, non thermalized particles, the Maxwellian distribution function is not completely adequate to describe the real distribution function of the species and the results can be affected by this approximation [32,33,34,35]. Nevertheless, as shown in past studies, the equivalent Maxwellian distribution function is good enough, at least in similar cases, to study the effect of fast ions on thermal transport and to obtain a good approximation of the heat fluxes from the simulations $[17,36]$. Typical grid parameters in the nonlinear simulations are as follows: perpendicular box sizes $\left[L_{x}, L_{y}\right] \approx$ $[190,210] \rho_{s}$, phase-space grid discretization $\left[n_{x}, n_{y}, n_{z}, n_{v \|}, n_{\mu}\right]=[256,72,48,64,16], 0.03 \lesssim k_{y} \rho_{s} \lesssim 2.1$ and $0.034 \lesssim k_{x} \rho_{s} \lesssim 4.34$. The plasma parameters used as input in the simulations have been taken from discharge n. 73224 (CW $D$ plasma with $3.5+8 \mathrm{MW}$ of ICRH + NBI, only linear simulations), n. 90671 (ILW $D$ plasma with $2.5+1.5 \mathrm{MW}$ of ICRH+NBI), n. 90673 (ILW $D$ plasma with $6.5+5 \mathrm{MW}$ of ICRH+NBI), n. 91626 (ILW $\mathrm{H}$ plasma with $3.5+0.75 \mathrm{MW}$ of ICRH $+\mathrm{NBI}$ ) and n. 91710 (ILW H plasma with $4+8 \mathrm{MW}$ of ICRH $+\mathrm{NBI}$ ) at $\rho_{\text {tor }}=0.33$ and $t \approx 6 \mathrm{~s}$. Some important parameters are listed in Table 1 while the fast ions parameters are listed in Table 2. The effect of plasma impurities $(\sim 1 \% B e, \sim 0.3 \% N e, \sim 0.05 \% N i$ for the ILW discharges, $\sim 2 \% C$ for the $\mathrm{CW}$ discharges) has been neglected in the simulations. The effect of these impurities has been found negligible compared to the effect of fast ions in past studies of similar discharges [36]. For a detailed gyrokinetic analysis of the CW discharge 73224 see ref. [17, 34, 35].

A quasi-linear modelling of the ILW discharges has also been done using the trapped gyro-Landau fluid model TGLF in its most recent version (SAT1 saturation rule) $[37,38,39]$. The model has been tested in standalone mode and coupled into the ASTRA transport code [40,41]. With respect to the original TGLF SAT0 model, TGLF SAT1 features the zonal flow mixing, rather than shearing, as the primary saturation mechanism of both ion and electron scale turbulence, and captures cross-scale coupling and non-linear upshift of the critical $R / L_{T i}$. However the model presently cannot capture the non-linear fast ion stabilization described in [17]. Fast ions have not been included as kinetic species in our simulations with TGLF, due to the onset of instabilities not properly resolved at the standard resolution used for profile simulations, but they have been included in the simulations for their contributions to main ion dilution and pressure. The simulations are electromagnetic $\left(B_{\perp}\right)$, use Miller geometry and cover the range $0.05 \lesssim k_{y} \rho_{s} \lesssim 17$. In stand-alone mode, in order to compare with GENE simulations, no impurities have been included, whilst profiles simulations include two impurities as kinetic species $\left({ }^{3} \mathrm{He}, B_{e}\right)$ besides main ions and electrons. The spectral shift $E \times B$ model is used. For the profile simulations the boundary condition has been set at $\rho_{\text {tor }}=0.8$.

\section{Experimental observations}

Two main cases have been considered for the experimental analysis: the first case corresponding to $\sim 4 \mathrm{MW}$ of total power (referred as 'low heating power' case), and the second case corresponding to $12 \mathrm{MW}$ of total power (referred as 'high heating power' case). In the first case, a high stiffness is observed on the whole ion temperature 
profile, while, in the second case, a strong reduction of the ion temperature stiffness and of $q_{i, g B}$, while reaching higher values of $R / L_{T i}$, is observed in the region $r / a \lesssim 0.4$. This strong reduction of the turbulent transport is related, as shown in ref. $[17,35,36]$ and in section 6 , to the presence of a high fast ion pressure that, through electrostatic [35] and electromagnetic [17] effects, strongly stabilize the ITG instability.

\section{1 'Low heating power' case}

The core ion temperature is lower over the whole radial profile in $H$ plasmas (see Fig. 2a), the difference starting outside $\rho_{\text {tor }} \approx 0.8$. The shape of the core ion temperature profile does not show substantial differences between $H$ and $D$ plasmas. Fig. 3a shows the $q_{i, g B}$ vs $R / L_{T i}$ plot at $\rho_{t o r}=0.33$, where the black symbols refer to the low power discharges. Both $\mathrm{H}$ and $D$ plasmas generally align well, consistently with the gyro-Bohm scaling, but the consistency with gyro-Bohm for what regards the ion mass dependence is difficult to isolate, as the variation of $T_{i}$, the high ion stiffness and the uncertainties on $R / L_{T i}$, mask any possible deviations form the scaling due to the ion mass. No substantial differences in the $R / L_{T i}$ turbulent transport critical threshold or in the ion stiffness are visible between $H$ and $D$ plasmas outside error bars. A similar conclusion holds for the $q_{i, g B}$ vs $R / L_{T i}$ plot at $\rho_{t o r}=0.5$, which we have added for comparison in Fig. $3 \mathrm{~b}$.

\section{2 'High heating power' case}

In the 'high heating power' case, there is still a difference in the ion temperature coming from the plasma edge, but a difference between $D$ and $H$ plasmas has been observed also in the plasma core inside $\rho_{\text {tor }} \lesssim 0.4$. As can be seen in Fig. 2b, while in $D$ plasmas a steepening of $T_{i}$ is clearly visible, in $H$ plasmas this effect is much lower. This is also seen in Fig.3a (red symbols), where the characteristic reduction of ion stiffness with respect to the low power cases (black) due to fast ion stabilization, previously reported [17,36], is visible both in $D$ and $H$ plasmas, but a significantly lower reduction in $q_{i, g B}$ is observed in $H$ than in $D$ at similar $R / L_{T i}$, resulting in an apparent anti-gyro-Bohm scaling, in the sense that a decrease in $q_{i, g B}$ is seen when moving from $\mathrm{H}$ to $D$ at same $R / L_{T i}$. On the contrary, at $\rho_{\text {tor }}=0.5$ (Fig.3b), where fast ion stabilization is not expected due to the central localization of ICRH fast ions and to the higher value of magnetic shear [42, 43], there is no reduction in stiffness at high power compared to low power, and hydrogen and deuterium points do not split, similarly to the case at low power. The origin of the anti-gyro-Bohm behavior at $\rho_{\text {tor }}=0.33$ at high power was the object of a detailed analysis using gyro-kinetic GENE simulations. Considering that there are no substantial changes in other plasma parameters, we studied the different effect of fast ions in these discharges. The question is whether the ITG stabilization related to fast ions is less effective in $H$ plasmas, or whether, more trivially, there is a substantial difference in the fast ion pressure between the $\mathrm{H}$ and $D$ plasmas.

A comparison between the fast ion pressure in $D$ and $H$ plasmas calculated by PION for ICRH and PENCIL for NBI is shown in Fig. 4. The fast ion pressure is found lower in $H$ plasmas. For ${ }^{3} \mathrm{He}$ this is due to the fact that the ion heating in $\mathrm{H}-\left({ }^{3} \mathrm{He}\right)$ plasmas is obtained using a ${ }^{3} \mathrm{He}$ concentration $\sim 2 \%$, while in $\mathrm{D}-\left({ }^{3} \mathrm{He}\right)$ plasmas it is obtained using ${ }^{3} \mathrm{He} \sim 6 \%$. This difference in the ${ }^{3} \mathrm{He}$ density, the ${ }^{3} \mathrm{He}$ temperature being comparable, brings to lower ${ }^{3} \mathrm{He}$ pressure in $\mathrm{H}$ plasmas. For NBI fast ions, both the fast ion density and temperature are lower in $H$ plasmas for a given level of heating power. These differences can be understood considering different factors. Firstly, the typical voltage used to accelerate the neutral beams was lower for the $H$ beams $(\sim 70 k V)$ compared with the $D$ beams $(\sim 100 k V)$, and a larger fraction of the $D$ beam power was injected at full energy $(\sim 52 \%)$ while the full energy component of the $H$ beams was just $\sim 31 \%$. This resulted in comparable average beam velocities in the two cases, but a much higher average energy in the case of the $D$ beams (by up to a factor of 2) and a correspondingly higher particle injection rate for the $H$ beams at a given level of heating power. Secondly, the average time for a beam injected fast ion to slow-down to the thermal velocity was much higher for the $D$ beams compared with the $H$ beams (by about a factor of 3). These two factors largely explain 
why the number of NBI fast ions in $D$ plasmas was significantly higher than in $H$ plasmas for a given heating power level, and why these fast ions had a higher average energy. It should also be noted that more off-axis beams were selected for the $H$ plasmas than the $D$ plasmas in these experiments, resulting in a broader NBI deposition profile. All these effects, even if the ion heating power profiles was similar, caused the differences in the total fast ion pressure between $H$ and $D$ plasmas shown in Fig. 4.

\section{Gyrokinetic simulations}

The linear gyrokinetic simulations indicate that, in all the considered cases, the ITG modes are the dominant modes at $\rho_{\text {tor }}=0.33$ and up to $k_{y} \rho_{s} \sim 0.8$. The linear simulations of the fast ion effect on the ITG modes for the 3 different discharges of the 'high power case' (using the values listed in Table 8 for the fast ion species) are shown in Fig. 5. For each discharge, a comparison is shown between the growth rate without fast ions and with fast ions, using both sets of fast ion parameters, i.e. in $D$ shots also the fast ion parameters of the H-91710 case, and in the $H$ shot also those of the D-90673 case. It is clear that the lower fast ion pressure of the $H$ plasmas has a lower linear stabilization effect with respect the higher fast ion pressure in the $D$ plasmas, irrespective of the different other parameters of the 3 discharges. Considering the values at $k_{y} \rho_{s}=0.25$, where the ion heat flux spectra have the maximum in the non-linear simulations, the ITG linear growth rate is reduced by $\sim 30-45 \%$ using the fast ion pressure of the $D$ plasmas, while it is reduced by only $\sim 15-20 \%$ using the fast ion pressure of the $H$ plasmas.

The ion heat fluxes obtained from non-linear gyrokinetic simulations are shown in Fig. 6a for the 'low heating power case' and in Fig. 6b for the 'high heating power case'. A good match is obtained in both cases. In the 'low heating power case' the simulations indicate a difference between the ion heat flux stiffness in $D$ and $H$ plasmas, caused mainly by the differences in the input parameters (such as $T_{e} / T_{i}, R / L_{n}$ and $R / L_{T e}$ ) rather than by different isotope mass. This can be seen from the simulation with hydrogen ions but using the same input parameters as the deuterium case (blue right-faced triangle with red contour in Fig. 6a, made at $R / L_{T i}=5.5$ where the discrepancy between the two cases is the highest), that gives almost the same ion heat flux as the one obtained in deuterium, indicating that no strong deviation from the gyro-Bohm scaling is expected in these plasma conditions. Experimentally, in Fig.3 the uncertainties are such that the differences introduced by slightly varying plasma parameters cannot be resolved and we can state that all the points are consistent with gyro-Bohm scaling at low power. In the 'high heating power' case, where an apparent anti-gyroBohm behavior is seen experimentally, the experimental fluxes are well reproduced when considering the effect of fast ions in electromagnetic simulations. With no fast ions the normalized ion heat fluxes are over-predicted by a factor 2 in $H$ and by a factor 4 in $D$. If in the simulation of the $D$ plasma the fast ion pressure of the $H$ plasma is used instead of the nominal one, the reduction of the ion heat flux is half of the one obtained using the fast ion pressure of $D$ plasmas and the flux ends up similar in value to that of the $H$ case. Consistently with the linear simulations, this result shows that the main reason for the apparent anti-gyro-Bohm behavior in the core of the high power cases is the different fast ion stabilization, which is caused by the difference in the fast ion pressure between $H$ and $D$ plasmas, due to different heating power settings and schemes. In these cases, other mechanisms that may also break the gyro-Bohm scaling have a minor role with respect to the different fast ion stabilization, as indicated by the fact that we get similar normalized fluxes when we use the $H$ fast ion population in the $D$ case.

\section{Quasi-linear analysis}

Stand-alone TGLF SAT1 simulations have been run for the 4 discharges at $\rho_{\text {tor }}=0.33$ to compare with the GENE results, and are shown in Fig. 6. Input parameters were identical to the GENE ones apart from the 
temperatures of the fast ion species. The low power shots had only the main ion and electron species both in GENE and TGLF, whilst for the high power shots in TGLF the ${ }^{3} H e$ was treated kinetically but at thermal temperature, whilst the NBI fast ions were only considered for dilution and pressure. This is the nearest configuration to the GENE run, being unable to treat kinetically the fast ion species with TGLF, whilst GENE treats both the ${ }^{3} \mathrm{He}$ ions and the NBI fast ions kinetically as two additional species. From Fig.6 it is evident that the TGLF ion stiffness is much lower than the GENE ion stiffness in the low power shots. This has already been observed in previous studies [44, 45, 46, 47]. In the high power shots, when including fast ions, the GENE stiffness drops significantly. As a result, the TGLF and GENE stiffnesses come to a (fortuitous) agreement in the $D$ case, whilst in $H$ due to the lower fast ion pressure the GENE stiffness remains higher than the TGLF one. This underestimate of the ion stiffness was one motivation for a revision of the TGLF model that is presently ongoing [48]. According to these findings from the stand-alone runs, one would expect that profiles simulations will overestimate temperatures in the low power discharges, both in $D$ and $H$, and more relevantly in $H$ where the stiffness discrepancy is larger. Instead, we can expect that the high power discharge in $D$ will be reasonably matched by TGLF, due to the compensation between the inherent low stiffness and the lack of non-linear fast ion stabilization effects, whilst the high power discharge in $H$ will again be overestimated. The results of TGLF profile simulations for the 4 discharges are shown in Fig. 7 and indeed confirm the expectations. For the low power shots a marked over-prediction of the temperature profiles, and particularly $T_{i}$, is observed over the whole profile both in $D$ and $H$ plasmas, and more marked in $H$, where also the density peaking is over-predicted. For the high power shots, the $D$ case is well reproduced, although there is still a mismatch in temperature peaking inside $\rho_{\text {tor }}=0.2$. However, the $H$ case at high power is again over-predicted, both temperatures and density, as in the $H$ low power case.

\section{Discussion and conclusions}

Differences have been observed between JET L-mode $H$ and $D$ plasmas with the same operational settings (heating power, geometry, $\left.I_{p}, B, q, n_{e}\right)$. Both $T_{e}$ and $T_{i}$ are lower in $H$ plasmas, the difference starting at the plasma edge. To gain insight into the core transport mechanisms, ICRH ion heating applied on- and off-axis has allowed to reconstruct the local $q_{i, g B}$ vs $R / L_{T i}$ plot at different radial positions. We do not observe differences in critical gradient threshold between $H$ and $D$, but rather in ion stiffness, depending on power level and radial position. In low power plasmas, the ion heat flux in the plasma core does not deviate from the gyro-Bohm scaling outside error bars, but, due to the high stiffness and to the stronger weight of $T_{i}$ with respect to mass in the gyro-Bohm normalization, it is difficult to make a certain conclusion on the mass dependence from our data. A difference in the ion heat flux has been instead observed at higher power at $\rho_{\text {tor }}=0.33$, in the situation where a strong stabilization of ITG modes by fast ions is expected. In this case, the stabilization of the ion heat transport is less strong in $H$ plasmas with respect to $D$ plasmas, introducing an apparent anti-gyro-Bohm mass scaling. GENE non-linear simulations indicate that the explanation of this difference is the difference of the fast ion pressure in $H$ and $D$ plasmas, in $H$ plasmas being $\sim 1 / 2$ of the one in $D$ plasmas when high NBI or ICRH heating is applied. This is mainly due for NBI fast ions to the lower beam injection energies and shorter beam slowing down time in $H$ plasmas and for the ICRH fast ions to the lower ${ }^{3} H e$ concentration needed for the ICRH minority scheme heating in $H$. Therefore, this is not an "inherent" isotope dependence of transport, but due to differences in the heating schemes in the two species. In our cases it is the strongest effect, and minor differences such as due to $E \times B$, collisions, etc. lie within experimental uncertainties. In outer regions $\left(0.5<\rho_{\text {tor }}<0.8\right)$, where fast ion effects become negligible, the situation is similar to the low power case, with stiff ions and no evident deviations from gyro-Bohm. A mention is due to even more external regions, $\rho_{\text {tor }}>0.8$, where differences in temperatures are systematically observed, with the $H$ plasma edge colder than the $D$ plasma edge. This region is characterized by different types of instabilities and a dedicated 
study is beyond the scope of this paper.

Whilst the gyro-kinetic simulations account completely for the experimental observations, TGLF SAT1 simulations show a number of issues. Our results in these experimental conditions suggest that the present version of TGLF leads to under-prediction of ion heat transport in all cases where ion stiffness matters, i.e. in conditions sufficiently above the ion critical gradient threshold, with increasingly worse performance with increasing amount of ion heating with respect to electron heating applied (see [49] for an example of a good TGLF simulation of a JET L-mode in $H$ and $D$ with low ion heating, unlike the ones discussed in this paper, which have strong ICRH ion heating). This under-prediction of ion heat transport above threshold holds unless non-linear e.m. stabilization is important, in which case there may be agreement but due to fortuitous compensation between the low TGLF stiffness and lack of non-linear e.m. stabilization. In particular, it seems that this stiffness underestimate is stronger in $H$, which may be at the origin of the generally worse performance of the model in $H$ plasmas. This may open the possibility that once the ion stiffness issue is cured in the model, it may become equivalently good in $H$ and $D$ plasmas. Of course then a way to properly account for non-linear e.m. stabilization should also be introduced in the quasi-linear model, to reach the agreement with GENE on the basis of the same physical mechanisms. We remark that these considerations on the TGLF model become irrelevant in all cases that are near marginal stability, where only the critical gradient threshold matters, as is often the case for high temperature H-modes, where the model is generally found to reproduce well the experimental profiles $[45,50]$. However, we also note that high power $\mathrm{H}$-modes may also have an important fast ion component, in which case stiffness may again come into play, so that the reliable application of the model has to be judged case by case, until an improved version fixing these issues will become available.

Thinking about the future $T$ and $D T$ campaigns at JET, some considerations can be made. In $T$, a concentration of $4 \% \leq{ }^{3} \mathrm{He} \leq 6 \%$ is expected to have the $80-90 \%$ of the power deposited on the ions, giving similar fast ions content as in $D$ discharges. Regarding the NBI beam, similar voltages as the ones used in $D$ will be used for the $T$ beam, giving similar beam energies but a lower beam velocity in $T$ with respect to $D$ beams. The slowing down time is lower in $T$, so a higher NBI fast ion pressure can be expect in $T$ at least for discharges with densities similar to the ones of the discharges studied in this paper. For higher density discharges, such as H-modes, the different beam penetration could bring to a different situation. In general, fast ions can play an important role for the stabilization of the turbulent ion heat transport in the plasma core (and might play a role also in ITER [51]) and they can contribute significantly to the total plasma pressure. To conclude it is important, when it is expected to play a role, to consider the effect of fast ions when studying the effect of the isotope mass on the on the turbulent transport and, when planning an experiment, to consider all the aspects that can influence the fast ion content in the plasma (such as the slowing down time) in order to have the best optimization of heating power/temperatures in different isotope plasmas.

\section{Acknowledgement}

The authors are thankful to Alessandro Di Siena and Michele Romanelli for precious discussions and suggestions. We acknowledge the CINECA award under the ISCRA initiative, for the availability of high performance computing resources and support. Part of the simulations presented in this work were performed at the COBRA HPC system at the Max Planck Computing and Data Facility (MPCDF), Germany. This work has been carried out within the framework of the EUROfusion Consortium and has received funding from the Euratom research and training programme 2014-2018 and 2019-2020 under grant agreement No 633053. The views and opinions expressed herein do not necessarily reflect those of the European Commission. 


\section{References}

[1] M. Bessenrodt-Weberpals et al., Nucl. Fusion 33, 1205 (1993)

[2] S. D. Scott et al., Phys. Plasmas 2, 2299 (1995)

[3] C. W. Barnes et al., Phys. Plasmas 3, 4521 (1996)

[4] C. F. Maggi et al., Plasma Phys. Control. Fusion 60, 014045 (2018)

[5] F. Ryter et al., Nucl. Fusion 49, 062003 (2009)

[6] Saibene G. et al., Nucl. Fusion 39, 1133 (1999).

[7] G. Manfredi, M. Ottaviani, Phys. Rev. Lett. 79, 4190 (1997)

[8] P. A. Schneider et al., Nucl. Fusion 57, 066003 (2017)

[9] J. Garcia et al., Nucl. Fusion 57 (2017) 014007

[10] M. Nakata et al., Phys. Rev. Lett. 118, 165002 (2017)

[11] B. D. Scott, Phys. Fluids B 4, 2468 (1992)

[12] P. Manas et al., accepted by Nucl. Fusion, 2018

[13] Xu Y. et al., Phys. Rev. Lett. 110, 265005 (2013).

[14] Garbet X. and Waltz R. E., Phys. Plasmas 3, 1898 (1996).

[15] ITER report n. ITR-18-003, ITER Organization, 2018.

[16] Van Eester D., et al., Plasma Phys. Control. Fusion 51, 044007 (2009).

[17] Citrin J. et al., Phys. Rev. Lett. 111, 155001 (2013).

[18] L.-G. Eriksson, T. Hellsten and U. Willén, Nucl. Fusion 33 (1993) 1037

[19] T. Hellsten and L. Villard, Nuclear Fusion 28, 285 (1998).

[20] Hedin J. et al., Nuclear Fusion 42, 527 (2002).

[21] Hellsten T. et al., Nuclear Fusion 44, 892 (2004).

[22] Challis C., et al., Nucl. Fusion 29, 563 (1989).

[23] Brix M. et al., Review of Scientific Instruments 79, 10F325 (2008).

[24] Wesson J. and Campbell D. J., 'Tokamaks', Oxford University Press, 4th edition (2011)

[25] Grierson B. A. et al., Rev. Sci. Instr. 83, 10D529 (2012).

[26] Delabie E. et al., Plasma Phys. Control. Fusion 52, 125008 (2010).

[27] Jenko F., et al., Phys. Plasmas 7, 1904 (2000).

[28] Görler T., et al., J. Comput. Phys. 230, 7053 (2011).

[29] E. A. Frieman and Liu Chen, Physics of Fluids 25, 502 (1982). 
[30] Brizard A. J., Hahm T. S., Rev. Mod. Phys. 79, 421 (2007).

[31] Miller R. L., et al., Phys. Plasmas 5, 973 (1998).

[32] C. Angioni and A. G. Peeters, Phys. Plasmas 15, 052307 (2008).

[33] G. J. Wilkie et al., J. Plasma Physics 81, 905810306 (2015).

[34] A. Di Siena et al., J. Phys. Conf. Ser. 775, 012003 (2016).

[35] A. Di Siena et al 2018 Nucl. Fusion 58054002

[36] N. Bonanomi et al 2018 Nucl. Fusion 58056025

[37] Staebler G.M. et al 2005 Phys. Plasmas 12102508

[38] Staebler G.M. et al 2007 Phys. Plasmas 14055909

[39] Staebler G.M. et al 2016 Phys. Plasmas 23062518

[40] G. V. Pereverzev, P. N. Yushmanov, Max-Planck-Institute fur Plasmaphysik, (2002).

[41] E. Fable et al., Plasma Phys. Control. Fusion 55, 124028 (2013).

[42] Mantica P. et al., Phys. Rev. Lett. 107, 135004 (2011).

[43] Citrin J. et al., Nucl. Fusion 54, 023008 (2014).

[44] P. Mantica et al., 58th Annual Meeting of the APS Division of Plasma Physics Volume 61, Number 18. October 31-November 4 2016, San Jose, California.

[45] P. Mantica et al., 23rd Joint EU-US Transport Task Force Meeting, 11-14 September 2018, Seville, Spain.

[46] N. Bonanomi et al 2018 Nucl. Fusion 58026028

[47] I. Erofeev et al., 2017 Nucl. Fusion 57126067.

[48] G. M. Staebler et al., 46th European Physical Society Conference on Plasma Physics, 8-12 July 2019, Milan, Italy.

[49] C. F. Maggi et al., submitted to Nucl. Fusion, 2019.

[50] C. Holland et al., 23rd Joint EU-US Transport Task Force Meeting, 11-14 September 2018, Seville, Spain.

[51] J. Garcia et al., Phys. Plasmas 25, 055902 (2018). 


\section{Tables}

\begin{tabular}{|c|c|c|c|c|c|c|c|c|c|}
\hline Shot \# & $s$ & $q$ & $T_{i} / T_{e}$ & $R / L_{n}$ & $R / L_{T e}$ & $R / L_{T_{i}}$ & $\beta_{e}$ & $\gamma_{E x B}$ & $\nu *$ \\
\hline \hline $90673(\mathrm{D}, \mathrm{ILW})$ & 0.5 & 1.55 & 0.9 & 2.2 & 6.2 & 6.5 & 0.0037 & 0.05 & 2.4 \\
\hline 91710 (H, ILW) & 0.5 & 1.7 & 1.0 & 2.4 & 6.9 & 6.0 & 0.0024 & 0.1 & 4.92 \\
\hline 90671 (D, ILW) & 0.4 & 1.78 & 0.8 & 3 & 6.3 & 5.5 & 0.0023 & - & 4.97 \\
\hline $91626(\mathrm{H}, \mathrm{ILW})$ & 0.5 & 1.7 & 0.65 & 1.3 & 4.8 & 5 & 0.0018 & - & 4.72 \\
\hline
\end{tabular}

Table 7.1: Main plasma parameters at $\rho_{\text {tor }}=0.33$ used as input in the gyrokinetic simulations. $\beta_{e}=8 \pi n_{e} T_{e} / B_{T}^{2}, \nu *=$ $\nu_{e i} \cdot q R_{0} / \epsilon^{1.5} c_{s}, \gamma_{E x B}=-r / q \cdot \partial \Omega_{t} / \partial r \cdot R_{0} / c_{s}$ where $\epsilon=r / R_{0}, \nu_{e i}=\sqrt{2} \pi Z^{2} e^{4} n_{i} \ln (\Lambda) /\left(\sqrt{m_{e}} T_{e}^{1.5}\right), \ln (\Lambda)$ being the Coulomb logarithm, and $v_{t h, e}=\sqrt{T_{e} / m_{e}}$.

\begin{tabular}{|c|c|c|c|c|c|c|c|c|c|}
\hline Shot \# & \multicolumn{4}{|c|}{ NBI $D$ or $H$ fast ions } & \multicolumn{4}{|c|}{${ }^{3} \mathrm{He}$} & Tot. \\
\hline & $n / n_{e}$ & $T / T_{e}$ & $R / L_{n}$ & $R / L_{T}$ & $n / n_{e}$ & $\bar{T} / T_{e}$ & $R / L_{n}$ & $R / L_{T}$ & $P_{\text {fast }} / P_{t h}$ \\
\hline $73224(\mathrm{D}, \mathrm{CW})$ & 0.06 & 9.8 & 14 & 3.1 & 0.07 & 7 & 1.49 & 14 & 0.45 \\
\hline $90673(\mathrm{D}, \mathrm{ILW})$ & 0.05 & 8 & 8.5 & 2.5 & 0.05 & 11.0 & 2.2 & 18 & 0.5 \\
\hline $91710(\mathrm{H}, \mathrm{ILW})$ & 0.04 & 7.5 & 10 & 2.0 & 0.022 & 10 & 2.4 & 25 & 0.28 \\
\hline
\end{tabular}

Table 7.2: Parameters for fast ions and ${ }^{3} \mathrm{He}$ species at $\rho_{\text {tor }}=0.33$ and used as input in the gyrokinetic simulations. In the 'low heating power' case, $P_{f a s t} / P_{t h} \approx 0.1$ and the fast ions are not considered in the simulations. 
Figures
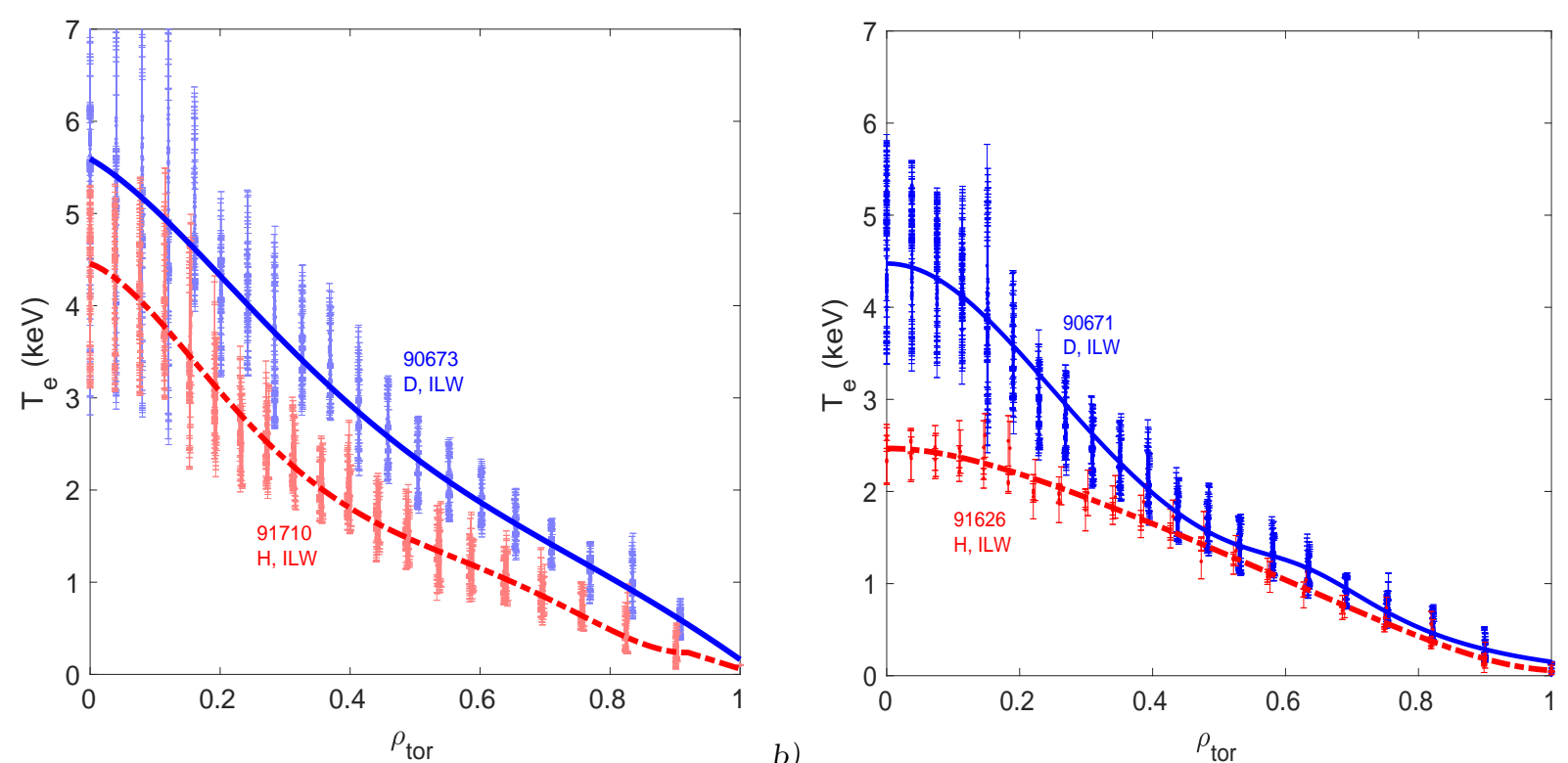

b)
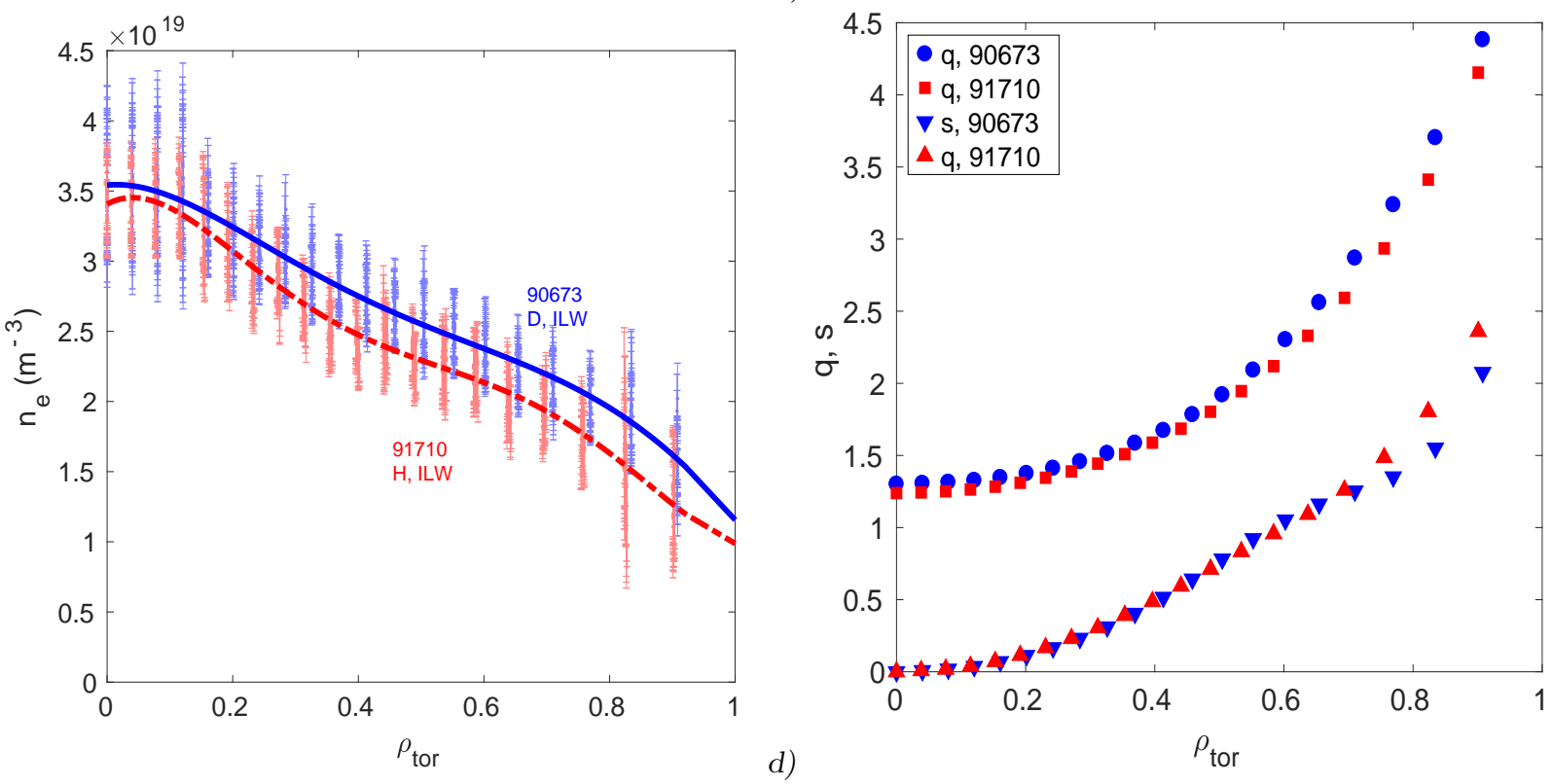

Figure 1: Electron temperature (a), electron density (c) and safety factor and magnetic shear (d) of 'high heating power' ILW discharges n. $90673(\mathrm{D})$ and n. $91710(\mathrm{H})$ and electron temperature $(\mathrm{b})$ of 'low heating power' discharges $n .91626$ (H) and $90671(D)$. 

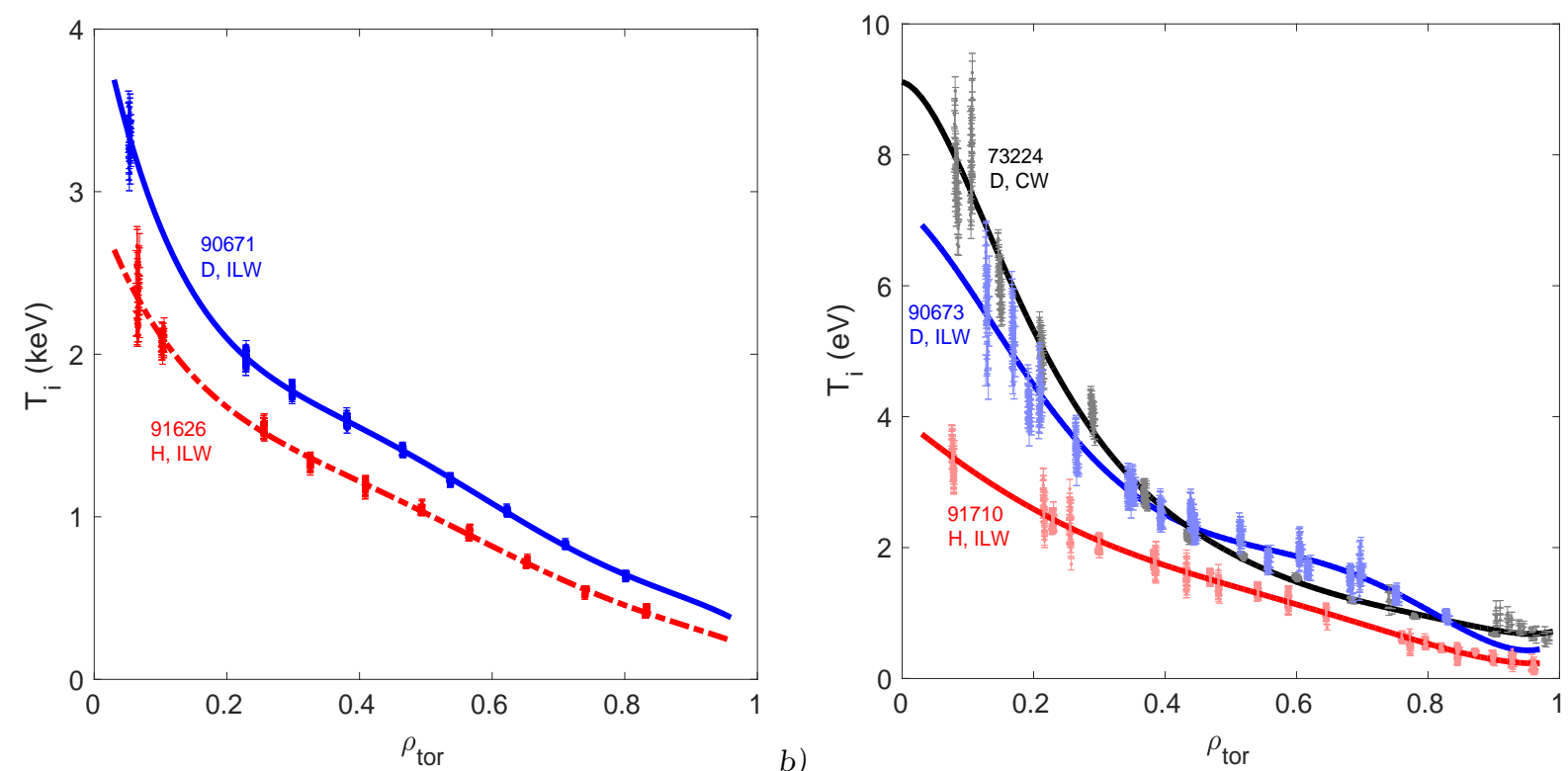

a)

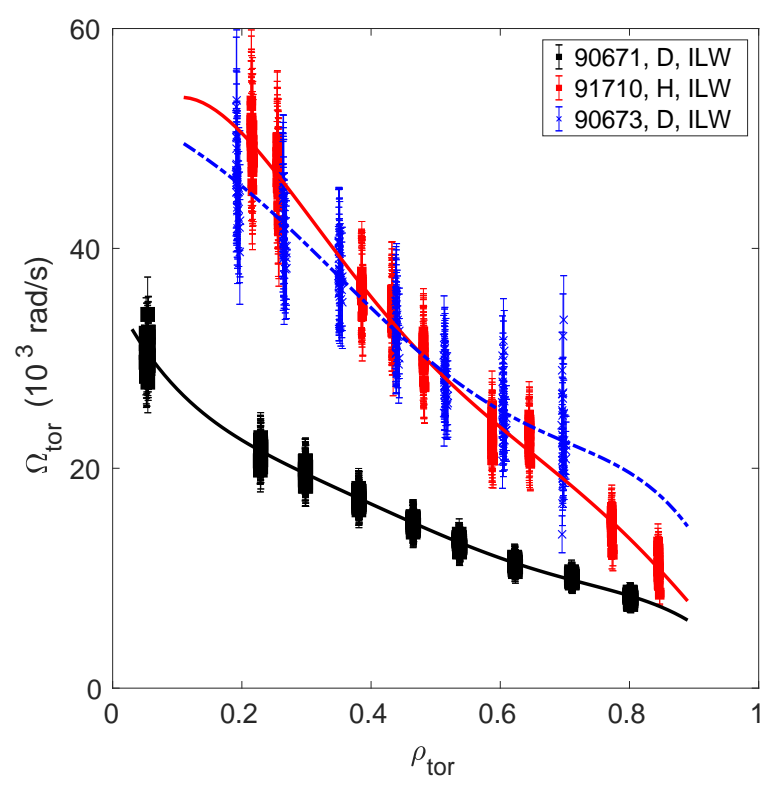

Figure 2: Ion temperature profile of (a) 'low heating power' ILW discharges n. 90671 (D) and n. $91626(H)$ and of (b) 'high heating power' discharges n. $91710(H), 90673(D)$ and 73224 (D, JET CW). c) Profiles of the plasma toroidal angular velocity. The toroidal angular velocity of shot $n .91626$ is very similar to the one of shot $n .90671$. 

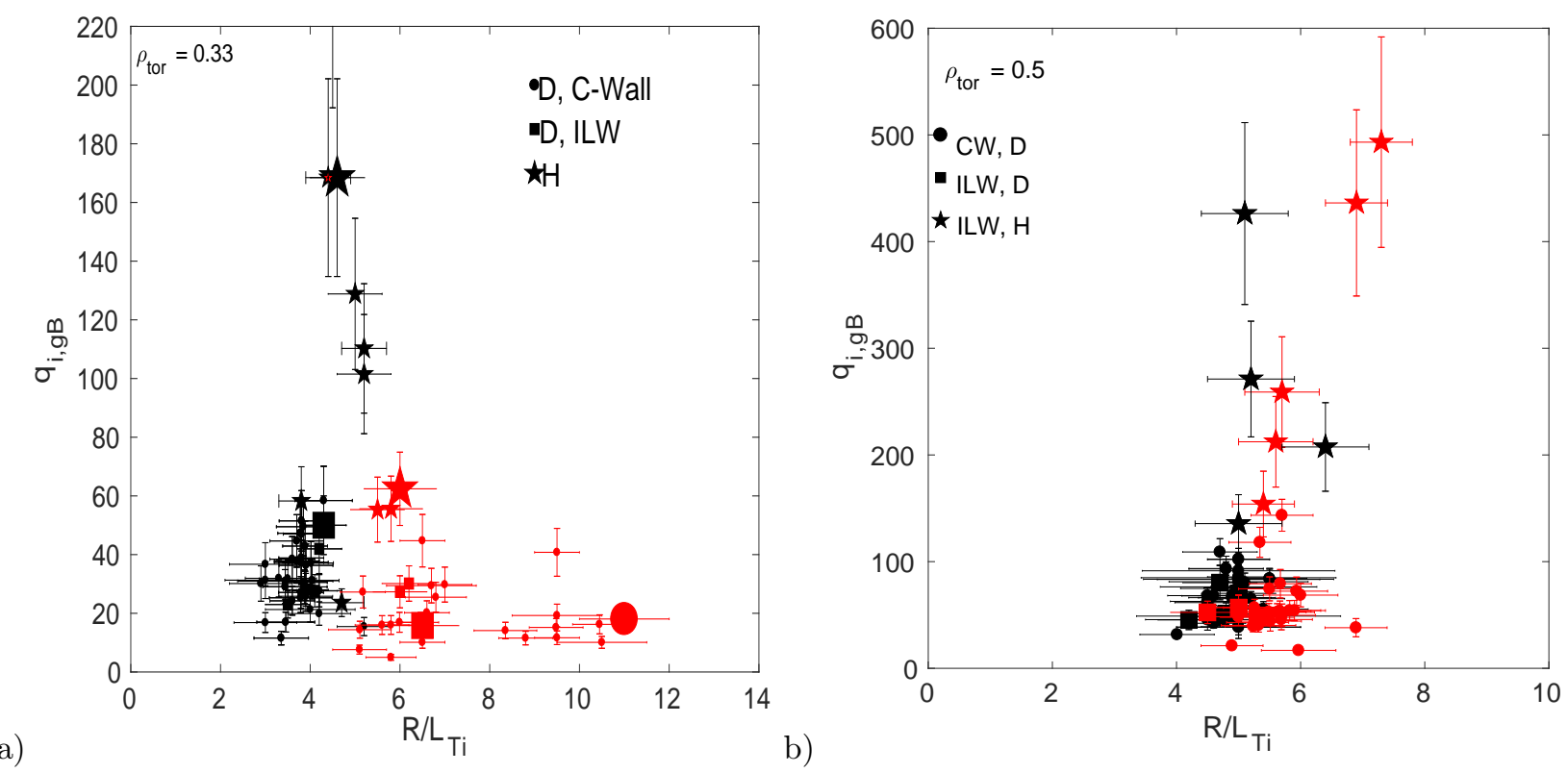

Figure 3: $q_{i, g B}\left(R / L_{T i}\right)$ at $\rho_{\text {tor }}=0.33$ (a) and at $\rho_{\text {tor }}=0.5$ (b) for a set of JET CW and ILW L-modes with similar $B_{T}, I_{p}, q_{95}$ and $n_{e 0} \sim 2.5-3.5 e^{19} \mathrm{~m}^{-3}$ but different levels of NBI and ICRH heating power. The black points correspond to the 'low heating power' case, while the red points correspond to the 'high heating power' case. The heat flux scans have been obtained using on- vs off-axis ICRH power. In figure (a) the plasmas considered in this paper have bigger points than the rest of the discharges.
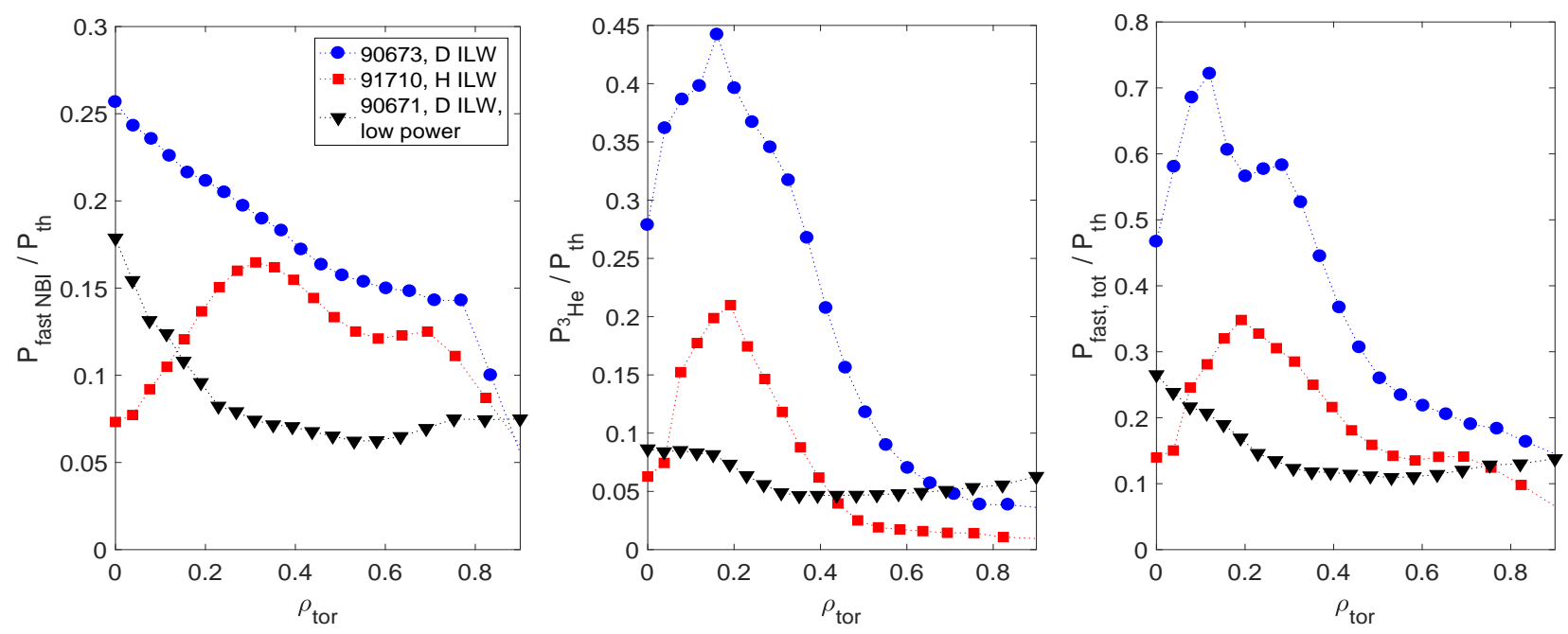

Figure 4: Pressure of the NBI fast ions (left, calculated using the PENCIL code), ${ }^{3} \mathrm{He}$ (center, calculated using the PION code) and total fast ion pressure (right) divided by the thermal species pressure (ions + electrons). 

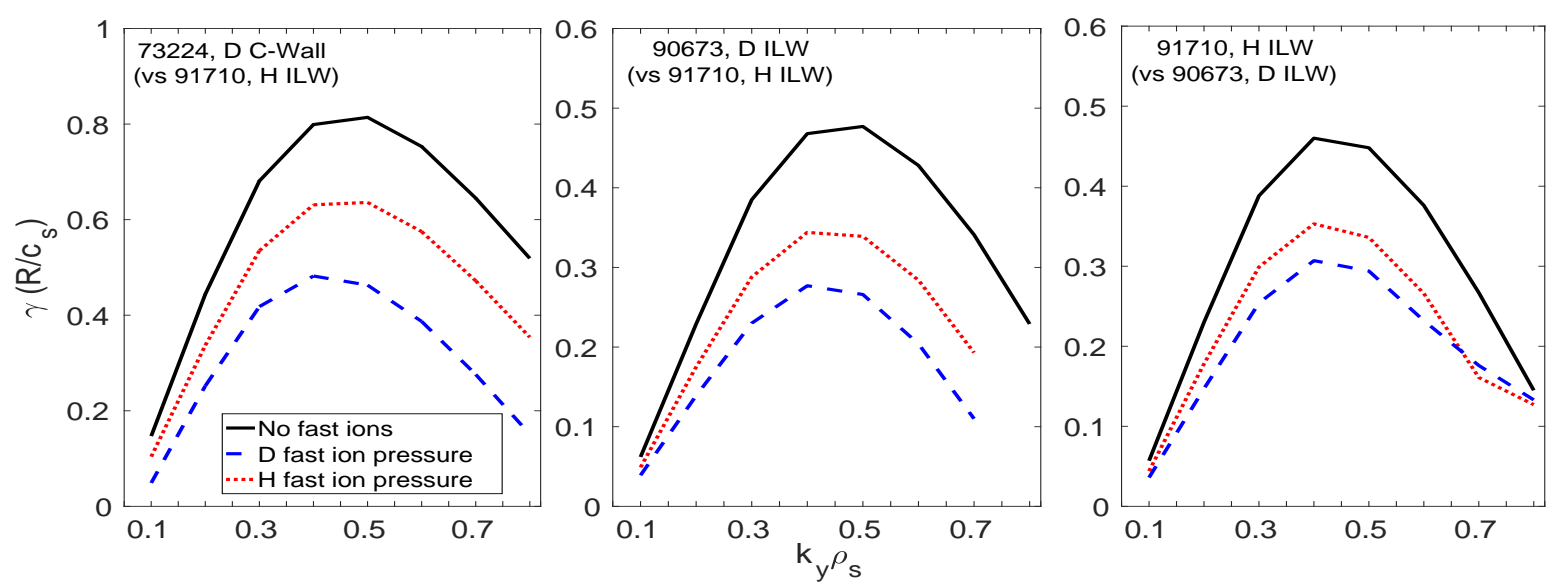

Figure 5: Comparison of the linear growth rate $\gamma \cdot\left(R / c_{s}\right)$ as a function of $k_{y} \rho_{s}$ without fast ions and with fast ions for discharges n. 73224, n. 90673 and n. 91710. For all the cases, a comparison with the effect of the different fast ion pressure in $D$ or $H$ plasmas is shown.
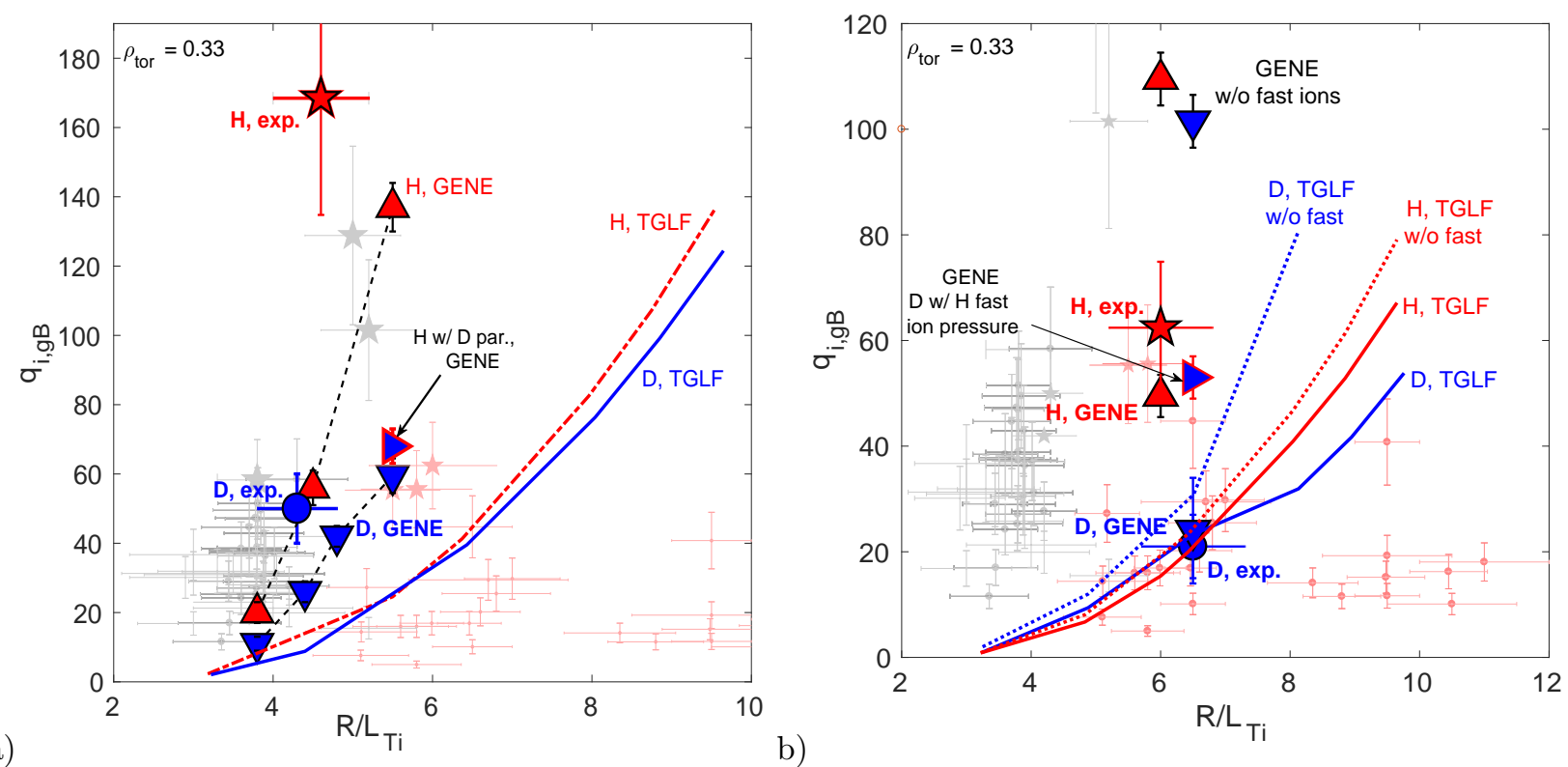

b)

Figure 6: Comparison between the experimental values of $q_{i, g B}\left(R / L_{T i}\right)$, the fluxes obtained from GENE non-linear simulations and the fluxes obtained from the TGLF-sat1 stand-alone simulations for the selected 'low heating power' case (a) and for the 'high heating power' case (b). In background the full data-set of Fig.3a is also shown. 

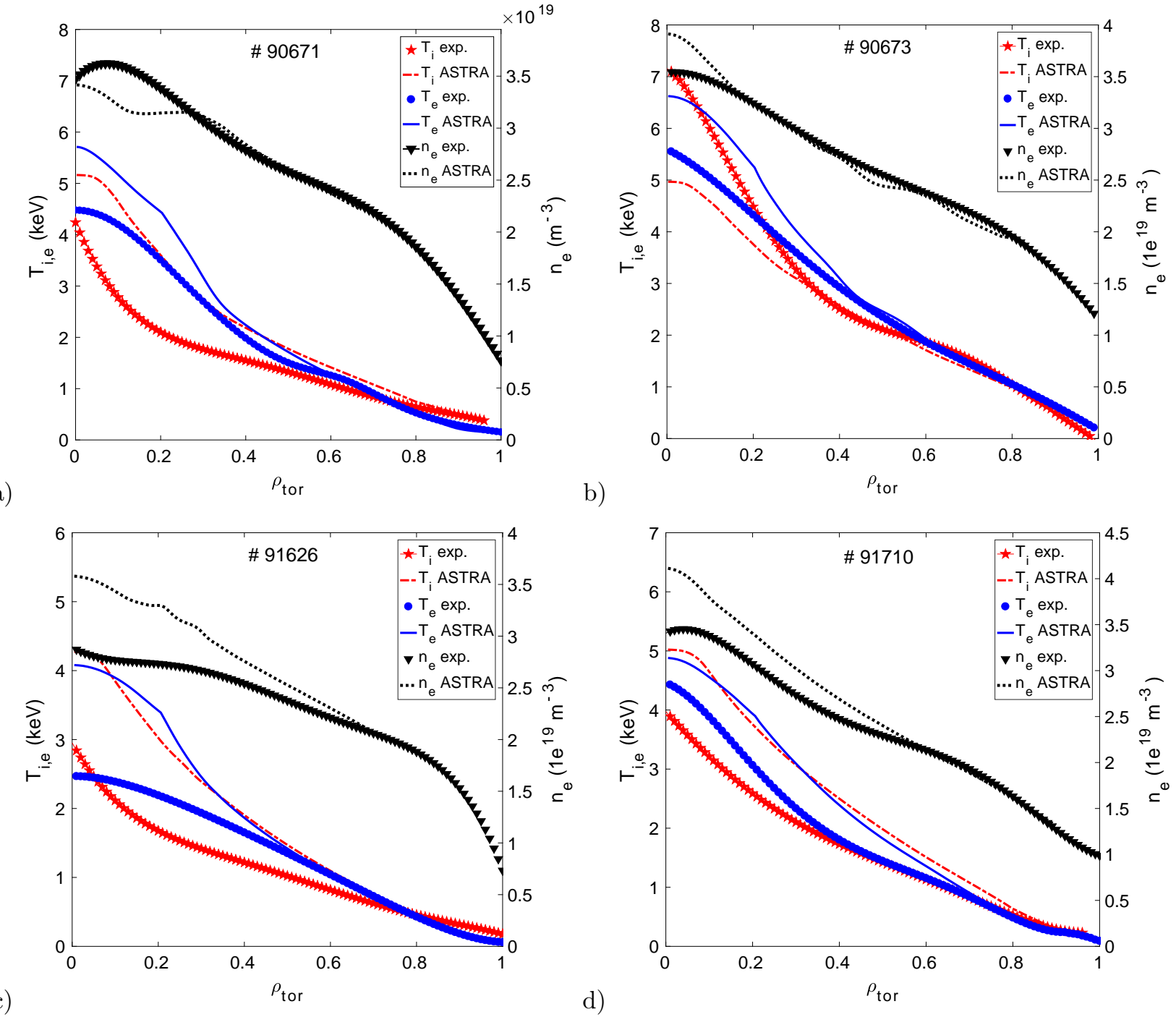

Figure 7: Comparison between the experimental profiles of $T_{i}$ (red pentagons), $T_{e}$ (blue circles) and $n_{e}$ (black triangles) and the profiles obtained from the ASTRA-TGLF simulations (solid blue line for $T_{e}$, dot-dash red lines for $T_{i}$ and dotted black lines for $n_{e}$ ) for the shots n. 90671 ( $D$ low power, plot (a)), n. 90673 (D high power, plot (b)), n. 91626 (H low power, plot (c)) and n. 91710 (H high power, plot(d)). 